\title{
Comparison of automated candidate gene prediction systems using genes implicated in type 2 diabetes by genome-wide association
} studies

\author{
Erdahl T Teber ${ }^{1}$, Jason Y Liu ${ }^{1}$, Sara Ballouz ${ }^{1}$, Diane Fatkin ${ }^{1,2}$ and \\ Merridee A Wouters*1,2
}

\begin{abstract}
Address: ${ }^{1}$ Victor Chang Cardiac Research Institute, 384 Victoria St, Darlinghurst, 2010, NSW, Australia and ${ }^{2}$ School of Medical Sciences, University of New South Wales, Sydney, Australia

Email: Erdahl T Teber - e.teber@victorchang.edu.au; Jason Y Liu - j.liu@victorchang.edu.au; Sara Ballouz - s.ballouz@victorchang.edu.au; Diane Fatkin - d.fatkin@victorchang.edu.au; Merridee A Wouters* - m.wouters@victorchang.edu.au

* Corresponding author
\end{abstract}

from The Seventh Asia Pacific Bioinformatics Conference (APBC 2009)

Beijing, China. 13-16 January 2009

Published: 30 January 2009

BMC Bioinformatics 2009, 10(Suppl I):S69 doi:10.1186/147I-2105-10-SI-S69

This article is available from: http://www.biomedcentral.com/I47I-2/05/I0/SI/S69

(C) 2009 Teber et al; licensee BioMed Central Ltd.

This is an open access article distributed under the terms of the Creative Commons Attribution License (http://creativecommons.org/licenses/by/2.0), which permits unrestricted use, distribution, and reproduction in any medium, provided the original work is properly cited.

\begin{abstract}
Background: Automated candidate gene prediction systems allow geneticists to hone in on disease genes more rapidly by identifying the most probable candidate genes linked to the disease phenotypes under investigation. Here we assessed the ability of eight different candidate gene prediction systems to predict disease genes in intervals previously associated with type 2 diabetes by benchmarking their performance against genes implicated by recent genome-wide association studies.

Results: Using a search space of 9556 genes, all but one of the systems pruned the genome in favour of genes associated with moderate to highly significant SNPs. Of the II genes associated with highly significant SNPs identified by the genome-wide association studies, eight were flagged as likely candidates by at least one of the prediction systems. A list of candidates produced by a previous consensus approach did not match any of the genes implicated by 706 moderate to highly significant SNPs flagged by the genome-wide association studies. We prioritized genes associated with medium significance SNPs.

Conclusion: The study appraises the relative success of several candidate gene prediction systems against independent genetic data. Even when confronted with challengingly large intervals, the candidate gene prediction systems can successfully select likely disease genes. Furthermore, they can be used to filter statistically less-well-supported genetic data to select more likely candidates. We suggest consensus approaches fail because they penalize novel predictions made from independent underlying databases. To realize their full potential further work needs to be done on prioritization and annotation of genes.
\end{abstract}




\section{Background}

The process of linking genes to disease phenotypes is rapidly gaining momentum since the first disease-causing gene was identified 25 years ago [1]. Alternative approaches adopted in the past to identify disease genes are the candidate gene approach, where likely suspects are prioritised and screened on a genome-wide basis; and linkage analysis where specific loci are determined systematically using family studies. The two approaches have been synthesized into a pipeline by completion of the Human Genome Project; and further enabled by the increased availability of high-throughput experimental data and the development of sophisticated bioinformatics tools. In addition there have been efforts in the bioinformatics community to systematize and automate candidate gene prediction. Automated prediction systems provide geneticists with a reduced list of genes estimated to have a high probability of involvement in the disease phenotype by sifting through hundreds to thousands of genes. Ultimately, these tools aim to give the researcher the best possible guidance in honing in on the gene culprits for further biological confirmation. Since their introduction in the early 2000s, the predictive powers of automated candidate gene prediction systems have improved, largely due to increases in biological systems knowledge and more effective algorithms.

Candidate gene prediction systems vary in their approach and the data sources they draw on in generating predictions. These are summarised in Figure 1 and Table 1. Comparing the performance of these systems can be difficult because of the use of custom benchmark test sets by individual groups. Typically, benchmarking data is derived from genotype-phenotype information from the Online Mendelian Inheritance in Man (OMIM) database [2], but groups have used varying subsets of diseases. Several groups have tried to use standard benchmark sets [35], but these efforts have been limited. In addition, it is difficult to predict whether benchmarks which predominately contain data on well characterised diseases with Mendelian transmission patterns (i.e. dominant, recessive, X-linked) resulting from mutations in single genes [6] will be effective in predicting genes involved in less well characterised diseases, or in complex diseases.

A recent effort by Tiffin and colleagues [7] to identify candidate disease genes for the complex disease type II diabetes (T2D) and the related obesity trait predicted 12 genes in previously implicated chromosomal regions. The study also allowed a limited comparison of seven candidate gene prediction systems. Since that time two genomewide association studies (GWAs) on T2D undertaken by the Wellcome Trust Case Control Consortium (WTCCC) and the Genetics Replication and Meta-analysis Consortium (DIAGRAM) have been published $[8,9]$. GWAs are a powerful tool for identifying genetic variants linked to complex diseases because they are more sensitive than linkage studies to small to moderate effect size contributions from polygenic and oligogenic diseases. The data from these GWAs allow the assessment of the predictions made by Tiffin et al., as well as evaluation of the effectiveness of predictions made by the individual automated candidate gene prediction systems used in their study and our system, Gentrepid [4]. We assessed the candidate gene predictions systems' ability to select robustly supported genes from the GWAs and used them to filter noisy data from statistically less well supported genes to select favoured candidates.

\section{Results \\ Predictions}

All methods were given the starting set of 9556 genes mapped to chromosomal intervals implicated in T2D as assessed by Tiffin et al., except for POCUS which was run against a search space of 562 genes. The POCUS method was confined to the smaller search space because "poorly defined susceptibility regions or regions with questionable association with the disease are obscured by background noise" [7]. The number of candidate gene predictions made by the eight methods varied from two to 3093. POCUS generated the smallest number of candidates but neither of the two predictions matched genes in either the highly significant (HS) or medium-to-highly significant (MHWD) data sets. Other candidate gene prediction methods made considerably more predictions. The largest numbers of predictions were made by $G 2 D$ (3,093 candidate gene predictions) and $\operatorname{eVOC}(2,496$ predictions). These comprise almost one third and one quarter of the search space respectively. Thus neither of these methods prune the search space particularly well. Excluding POCUS, the least number of predictions was made by Gentrepid comprising 502 genes in known-disease-gene mode.

\section{Accuracy of predictions}

To assess the accuracy of the predictions, all eight systems were compared with genes found in previously-implicated intervals strongly linked to T2D by the GWAs. Figure 2 shows the comparative performance of seven of these methods in selecting the 11 genes in the HS GWA data set. Several metrics were calculated to assess accuracy. No metrics were calculated for POCUS as neither of its two predictions matched genes in either the HS or MHWD data sets.

The Enrichment Ratio is a general measure of the system's ability to accurately prune the search space. Enrichment Ratios ranged from 1 to 5 for the seven remaining prediction systems. The highest Enrichments Ratios were obtained by Gentrepid and GeneSeeker. These results were robust when the upper and lower (not shown) 95\% 
Table I: Automated Candidate Gene Prediction Systems

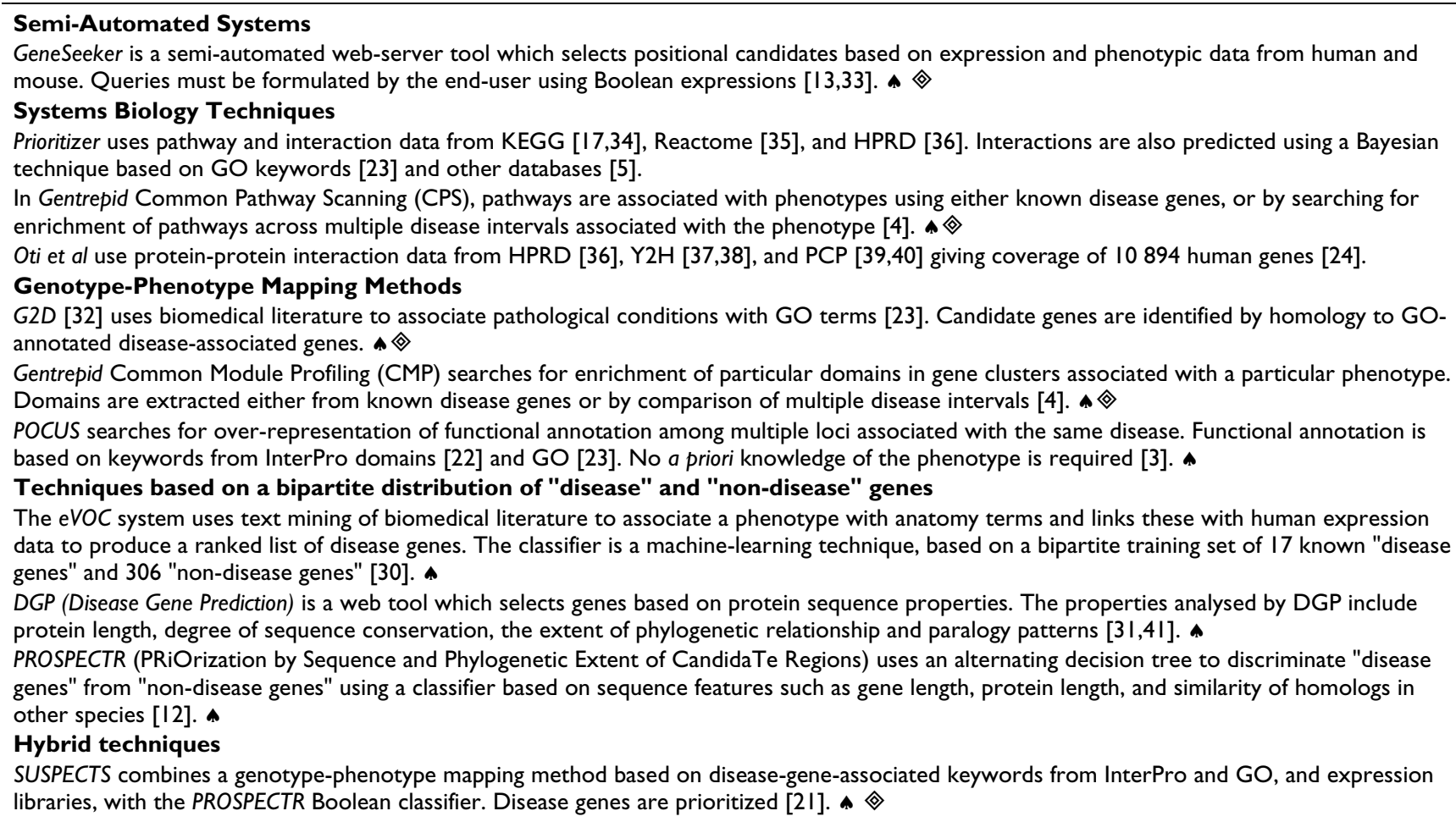

^ Assessed here, $\diamond$ Webserver.

confidence interval limits were taken into account. The lowest Enrichment Ratios were associated with the Machine Learning methods. This is not surprising, as the classifiers are trained to distinguish "disease genes" from "non-disease genes" and are ignorant of any concept of phenotype. The Specificity of a system measures its ability to reject genes not associated with the phenotype. Specificity scores among all seven methods ranged from 0.68 to 0.99 , with a median of 0.92 . As a group, the Machine Learning methods were poorer at rejection. G2D also performed poorly on this metric, but this result is slightly misleading because it does not take into account G2D's prioritization method which will be discussed later.

The Sensitivity is a measure of a system's ability to find the disease genes in the search space. A caveat here is not all of the GWA predictions are currently confirmed. G2D is by far the standout performer in Sensitivity, with $e V O C$ ranked second. However, as can be seen from the other metrics, this result is obtained at the expense of Specificity for both systems. Gentrepid's Sensitivity is on par with most of the Machine Learning methods but with higher Specificity. The high Specificity reflects the high quality of the data in the underlying databases. The lower Sensitivity is due to incompleteness of these databases with respect to all human genes.
Figure 2 shows the comparative performance of methods when assessed against the $61 \mathrm{~T} 2 \mathrm{D}$ associated genes with moderate to strong SNP signals (MHWD) in the Tiffin chromosomal intervals. The MHWD data set is not as statistically well supported as the HS set, and would be expected to contain some genes associated with T2D and others that are false positives. Perhaps the most interesting metric to look at here is the Sensitivity which should fall compared to the values for the HS set because of the lower signal to noise ratio in the MHWD set. All the systems except one, SUSPECTS, passed this negative test. More importantly, application of the systems to this noisy genetic data allows selection of a subset of candidates on the basis of molecular data (see below).

The results shown for Gentrepid in Figure 2 are for the known-disease-gene mode. In ab initio mode, Gentrepid's CPS method identified 506 pathways containing a total of 1980 candidate gene predictions. This resulted in Enrichment Ratios of 3.3 and 2.1 when the HS and MHWD full gene sets were considered (Table 2).

In ab initio mode, the CMP method generated 527 predictions by limiting the selection to the top $10 \%$ most probable genes. This resulted in correct prediction of one gene from the HS set and five from the MHWD set, yielding a 
A Data Sources

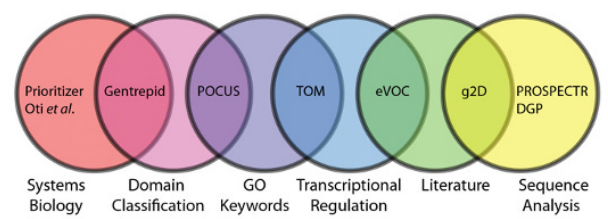

\section{B Approaches}
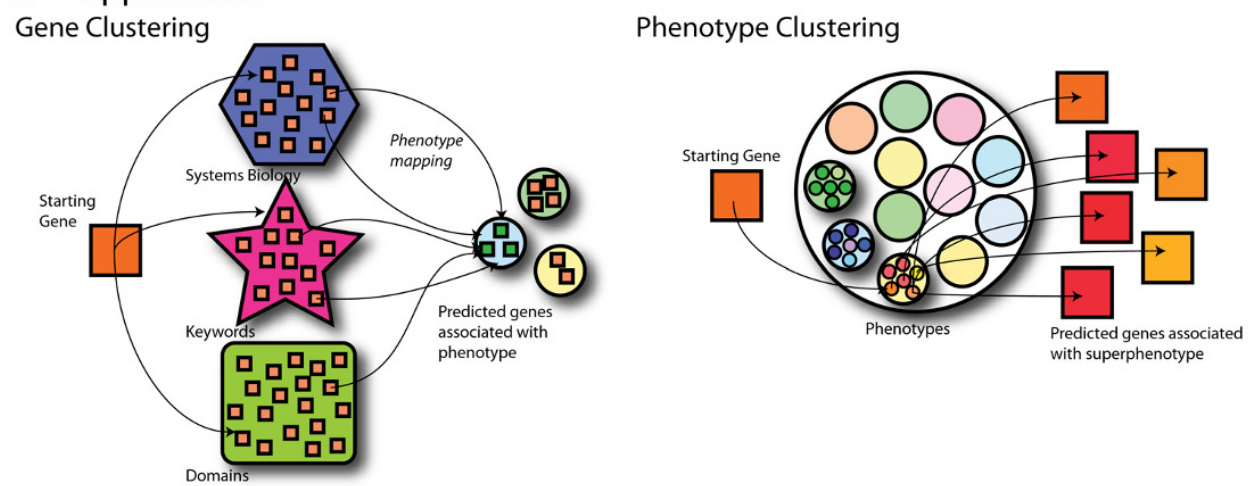

Machine Learning

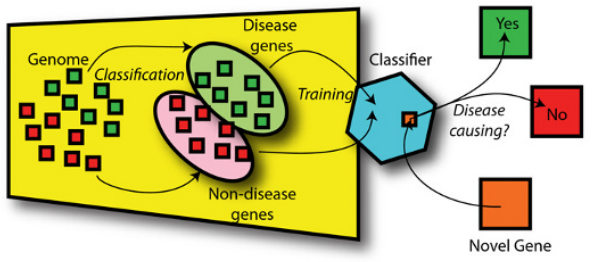

Transitive

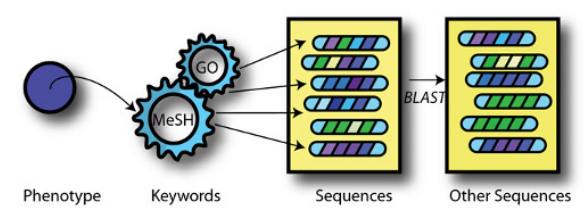

Figure I

Data sources and approaches used in automated candidate gene prediction methods. (A): Most systems draw on at least two types of data. SUSPECTS [2I] (not shown) uses keywords from InterPro [22] and GO [23], co-expression data, and also incorporates the PROSPECTR module [12] (shown on right). (B): Upper left Gene clustering approaches associate a gene cluster with a phenotype via a group member. For example, Systems Biology approaches [4,5,24] group genes whose protein products interact; and link them to a phenotype using a group-member gene associated with the phenotype. Systems Biology methods assume oligogenic diseases are associated with disruption in proteins that participate in a common complex or pathway [25]. Other gene clustering systems look for enrichment of keywords or domains associated with particular phenotypes and suggest candidate genes with similar properties. These systems are based on the principle that candidate genes have similar functions to disease genes already determined [26-28]. Upper right Phenotype clustering approaches such as that of Freudenberg \& Propping [29] group related phenotypes into superphenotypes. Lower left Most of the Machine Learning approaches do not use phenotype information and are based on the concept that the genome consists of a bipartite distribution of genes: those which cause diseases, and those that do not. By analysing these two gene sets with respect to discriminating variables, a profile for "non-disease genes" and "disease genes" is produced which enables training of a classifier. A novel gene submitted to the classifier is flagged as either "disease-causing" or "non-disease causing". Systems include eVOC [30], PROSPECTR [I2], SUSPECTS [2I] and DGP [3I]. Finally G2D, lower right, is a transitive method that maps phenotypes to genes [32] by interfacing literature- and keyword-based ontologies.

Enrichment Ratio of 2.2 when applied to the HS and 2.0 for the MHWD gene data sets.

It is also interesting to note the effect of lack of annotation on these results. Only five of 11 genes in the HS dataset, and 19 of 61 genes in the MHWD set contained KEGG or BioCarta pathway annotations. When we included only genes containing pathway information from the gene datasets (designated 'annotated' in Table 2) we observed Enrichment Ratios of 7.2 against the HS and 6.8 against the MHWD pathway-annotated sets. Sensitivities also improved by a factor of 2 for the HS dataset. By extrapolation, if all genes were pathway annotated, we could expect approximately two- to three-fold improvement in Enrichment and Sensitivity scores. 

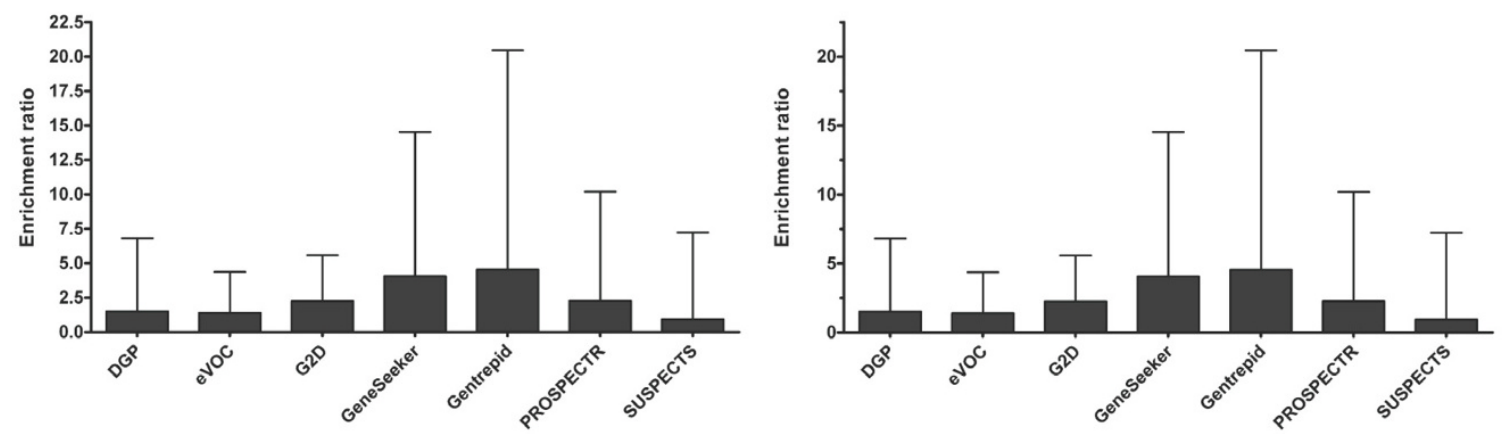

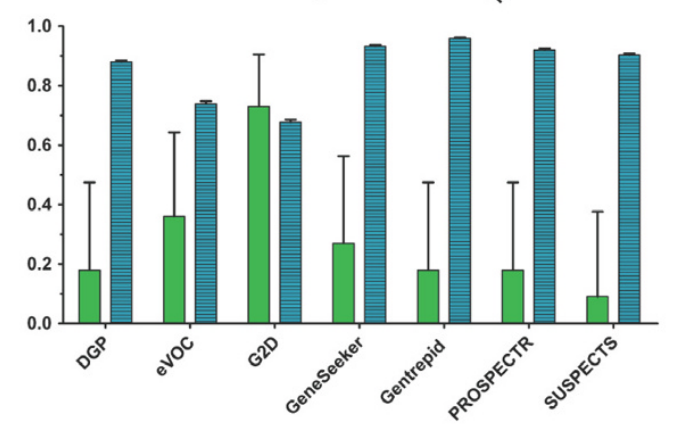

HS

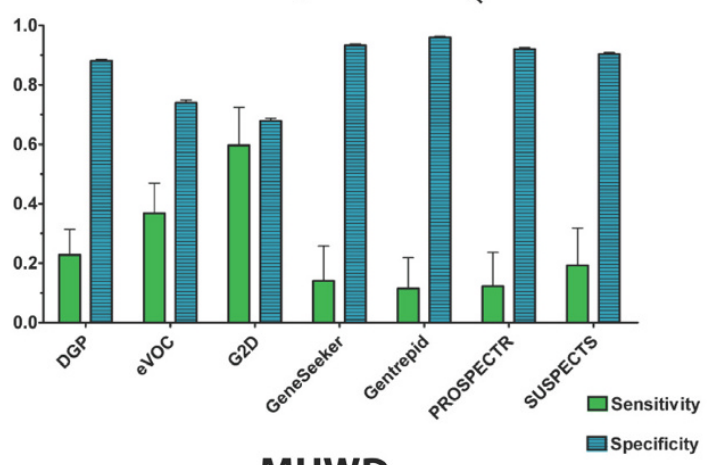

MHWD

Figure 2

Comparison of methods against the HS (left) and MHWD (right) T2D gene data sets. Top: Relative Enrichment Ratios. Bottom: Comparisons based on Sensitivity and Specificity.

\section{Prioritization}

Although the metrics discussed provide useful measures of a candidate gene prediction system's performance, another criterion of importance to geneticists is the system's ability to prioritize predictions. Although several methods claim the ability to prioritize (Table 1 ), only G2D provided prioritized predictions in Tiffin et al. [7]. Hence only G2D and Gentrepid will be discussed here. Because Gentrepid only made 502 predictions in toto, we took the top 502 predictions made by G2D and recalculated the Enrichment Ratio, Sensitivity and Specificity for this restricted set of favoured predictions. ER and Specificity significantly improved to 3.1 and 0.95 such that G2D surpassed Gentrepid's gross ER and almost equalled Gentrepid in Specificity. The improvement in these two metrics came at the expense of Sensitivity which was reduced to 0.16 , but the G2D system still managed to maintain its lead on this metric.

In G2D's prioritization system, a GO-metric is calculated for each gene in the search space based on how well its GO profile fits the GO profile of the disease genes inferred from MeSH terms. An R-score is calculated for each gene by normalizing against the number of genes in the genome with better GO-metrics for the phenotype. Genes with R-scores closer to zero are better fits to the phenotype.

Gentrepid CPS ranks genes by the number of loci in the search space involved in a particular pathway. In $a b$ initio mode, of the 53 intervals searched, the top pathway, focal adhesion, was represented in 35 of these. All five of the HS dataset genes were represented by pathways found in at least eight intervals. Pathways implicated in at least eight intervals constituted the top $40 \%$ of the 506 pathways containing 1749 candidates. In these pathways, Gentrepid identified all five pathway annotated genes from the HS dataset, and 18 of the 19 pathway annotated genes from the MHWD gene data set. Other figures for CMP are given in Table 2.

CMP $a b$ initio looks for protein domains enriched in the search space compared to the genome by taking a census of domains in the search space and the genome. Two expectation values are calculated to estimate the frequency of occurrence of genes with domains of interest based on a random combination of these domains $e_{a}$ and the rarest domain $e_{b}$ [4]. Figure 3 shows the data ranked on a $\chi^{2}$ test based on $e_{a}$ was most effective in prioritizing the HS data. This reflects our experience of phenotypes with genotypes 
Table 2: Gentrepid ab initio results

\begin{tabular}{|c|c|c|c|c|c|c|c|}
\hline Predictions & Reference list & ER & L95\% & U95\% & $\mathrm{S}$ & L95\% & U95\% \\
\hline CPS rank $8+$ pathways & HS & 3.3 & 1.1 & 9.4 & 0.45 & 0.21 & 0.72 \\
\hline CPS rank $8+$ pathways & HS - annotated & 7.2 & 2.1 & 25 & 1.00 & 0.57 & 1.00 \\
\hline CPS rank $8+$ pathways & MHWD & 2.1 & 1.3 & 3.6 & 0.30 & 0.20 & 0.42 \\
\hline CPS rank $8+$ pathways & MHWD - annotated & 6.8 & 3.6 & 13 & 0.95 & 0.75 & 0.99 \\
\hline CPS interactions top $50 \%$ & HS & 4.1 & 1.2 & 15 & 0.27 & 0.10 & 0.57 \\
\hline CPS interactions top $50 \%$ & HS - annotated & 9.0 & 2.2 & 37 & 0.60 & 0.23 & 0.88 \\
\hline CPS interactions top $50 \%$ & MHWD & 1.7 & 0.79 & 3.8 & 0.11 & 0.06 & 0.22 \\
\hline CPS interactions top $50 \%$ & MHWD - annotated & 8.1 & 3.2 & 20 & 0.54 & 0.29 & 0.77 \\
\hline CMP top $10 \%$ & $\mathrm{HS}$ & 2.2 & 0.3 & 17 & 0.1 & 0.02 & 0.38 \\
\hline CMP top $10 \%$ & MHWD & 2.0 & 0.8 & 4.8 & 0.1 & 0.08 & 0.18 \\
\hline
\end{tabular}

Abbreviations in Table: ER - Enrichment Ratio, L95\% - Lower 95\% confidence limit, U95\% - Upper 95\% confidence limit, S - Sensitivity

encoded by multi-domain proteins, as would be expected for diseases associated with signaling. For metabolic diseases associated with single-domain proteins, $e_{b}$ may be a better measure.

Although the G2D prioritization system appears more sensitive than the coarse-grained prioritization of Gentrepid, the performance of both systems was roughly equivalent against the HS set. Both systems were moderately successful in prioritizing the HS data. For example, of the seven genes in the HS dataset predicted by G2D, four were ranked in the top $15 \%$ by G2D's prioritization method (bold in Figure 3). Significant work needs to be done to improve the prioritization schemes of both G2D and Gentrepid.

Finally, we used the candidate gene predictions systems to filter the less statistically-well-supported MHWD data set (MHWD - HS): effectively adding more power to the GWA study. Prioritized predictions are the unbolded genes in Figure 3. Additional unprioritized predictions made for the MHWD dataset using the other candidate predictions systems are given as supplementary data in Additional file 1.

\section{Discussion}

Candidate disease gene prediction is a rapidly moving area of bioinformatics research with the potential to deliver great benefits to human health. By assisting geneticists to use existing biological information to investigate disease loci obtained by linkage analysis and association studies, disease genes can be identified more rapidly. The need for good applications in the area of candidate gene prediction is becoming increasing important as the proliferation of SNP-based association studies produces valuable genetic information in need of analysis.

The biggest problem facing candidate gene prediction today is the accuracy and completeness of the underlying databases. Failure to make a prediction is mostly due to incomplete data coverage. For example, $65 \%$ of human proteins have GO terms but only $25 \%$ of these are manually annotated. Systems drawing on GO terms like G2D are potentially able to make predictions for $65 \%$ of genes but only around one third of these are likely to be accurate. Systems Biology methods like Gentrepid CPS are reliant on pathway and protein-protein interaction data. One of the databases CPS draws on is OPHID [10], one of the most complete protein-protein interaction datasets, containing over 48000 interactions. However these 48,000 interactions are estimated to be only $13 \%$ of the complete human interactome [11]. Completeness of the underlying data clearly impacts the Sensitivity of the Gentrepid CPS method. As time goes on this constraint will ease as these databases are further populated. In the meantime, we have shown that the use of independent biological data to make complementary candidate gene predictions is one way to ameliorate the problem of incomplete data coverage (see Figure 3) [4].

In addition to the predictions made by the individual candidate gene prediction systems in Tiffin $e t$ al., a set of nine "winners" were chosen using a consensus approach [7]. These nine candidate genes were independently predicted by six of the seven prediction systems studied. A larger consensus set, chosen by five of the seven methods, contained 94 genes [7]. None of the genes in either of these consensus lists matched any of the genes in the HS and MHWD gene sets. Even if we compile a third tier of consensus genes from any four of the seven methods (269 genes) only one gene (VEGF) fell within the HS data set and only three genes (CHN2, B4GALT5, VEGF) matched the MHWD data set. Clearly the consensus approach is not working and it is easy to see why when the underlying databases are considered (Figure 1A). Candidate gene prediction systems that use an independent data set, not drawn upon by most of the other methods, will be penalized. Possibly the only benefit of a consensus approach is 


\begin{tabular}{|c|c|c|c|c|c|}
\hline \multicolumn{2}{|c|}{ G2D } & \multicolumn{4}{|c|}{ Gentrepid } \\
\hline & & \multicolumn{2}{|c|}{ CPS ab initio } & \multicolumn{2}{|c|}{ CMP ab initio } \\
\hline gene & rank & gene & rank & gene & rank \\
\hline XYLB & 137 & VEGF & $1-81$ & ZNF694 & $60-4$ \\
\hline ESR1 & 153 & LAMA1 & $1-81$ & CREB5 & $60-2$ \\
\hline VEGF & 185 & CX3CR1 & $82-371$ & BCL11A & $60-4$ \\
\hline IDE & 241 & ARHGEF12 & $82-371$ & ZNF532 & $60-4$ \\
\hline CEP110 & 267 & CCL18 & $82-371$ & $\begin{array}{l}\text { PTPRT } \\
\text { PT }\end{array}$ & $508-t$ \\
\hline CAMK1D & 276 & PDE4B & $531-608$ & ANKS1A & $520-6$ \\
\hline CHN2 & 373 & CDKN2B & $609-618$ & CX3CR1 & 853-11 \\
\hline ALS2CR19 & 435 & CDKN2A & $701-952$ & FSTL4 & 1109-1: \\
\hline HHEX & 479 & B4GALT5 & 701-952 & ESR1 & $1231-1:$ \\
\hline ZNF508 & 601 & CREB5 & $1009-1032$ & GPR133 & $1255-1:$ \\
\hline BCL11A & 677 & BLNK & $1067-1071$ & CAMK1D & $1372-14$ \\
\hline TSPAN8 & 752 & $A B C C 5$ & $1380-1459$ & RBMS1 & $1491-1$ : \\
\hline CREB5 & 761 & DARS2 & $1380-1459$ & COL22A1 & $1577-16$ \\
\hline SCN11A & 929 & HHEX & $1460-1541$ & ARHGAP26 & $1601-1 \epsilon$ \\
\hline ABCC5 & 952 & TRAC & $1542-1613$ & C14orf143 & $1680-1 ;$ \\
\hline PAX5 & 1213 & ESR1 & $1649-1699$ & ARHGEF 12 & $1773-1 \varepsilon$ \\
\hline CDKN2A & 1311 & UTRN & $1700-1751$ & ALS2CR19 & $1773-1 \varepsilon$ \\
\hline ANKS1A & 1351 & IDE & $1700-1751$ & HHEX & $1834-15$ \\
\hline CCL18 & 1472 & & & & \\
\hline UTRN & 1577 & Rankinaleaend & & & \\
\hline C6orf107 & 1603 & \multicolumn{2}{|c|}{ Top 250} & & \\
\hline $\mathrm{EBF} 3$ & 1653 & \multicolumn{2}{|c|}{ Between 251 to 500} & & \\
\hline C14orf143 & 1743 & \multicolumn{2}{|c|}{ Between 501 to 1000} & & \\
\hline $\begin{array}{l}\text { PTPRT } \\
\text { PT }\end{array}$ & & \multicolumn{2}{|c|}{ Between 1001 to 1500} & & \\
\hline CDKN2B & 1799 & \multicolumn{2}{|c|}{ Over 1500} & & \\
\hline
\end{tabular}

Figure 3

MHWD dataset filtered against prioritized automatic candidate gene predictions. Genes in bold are robustly supported genes from the GWA studies (HS set).

to give the user a false sense of accuracy when confronted with noisy data.

Clearly much work still remains to improve the sensitivity and specificity of candidate gene prediction methods but some general conclusions are possible. Machine Learning methods were not as effective as other methods. Most of the Machine Learning approaches do not use phenotype information and are based on the concept that the genome consists of a bipartite distribution of genes: those which cause diseases, and those that do not. The evidence supporting this assumption is limited [12]. We believe the concept that there is a difference between "disease genes" and "nondisease genes" is intrinsically flawed and no such Boolean classification exists. We hypothesize that the ability of these methods to predict disease genes in test sets is based on selection effects in the data: possibly rare, highly penetrant monogenic diseases, such as those involved in metabolic syndromes, are over-represented among known disease genes because they have been easier targets to identify. Although these systems were not as effective as the other candidate gene prediction systems, their performance was not greatly different. However, we believe that unlike systems which attempt to map genotype to phenotype, Machine Learning systems based on the disease gene/non-disease gene concept will not improve as more biological data becomes available.

\section{Conclusion}

Candidate gene prediction systems have typically been benchmarked on well characterized oligogenic phenotypes. GeneSeeker [13] produced a 10-fold enrichment using a data set consisting of eight diseases. Gentrepid's combined methods [4] produced an Enrichment Ratio of 13 when 29 diseases with a total of 170 known disease genes were used. For 29 diseases with 163 genes, POCUS [3] reported Enrichment Ratios between 12 to 42-fold, depending on the size of the intervals in the search space. The PRIORITIZER [5] method yielded a 2.8-fold enrichment using a data set consisting of 96 heritable disorders. In summary, Enrichment Ratios of 3 to 13 have been reported in benchmarks, but a substantial part of the data used for these studies has been limited to oligogenic phenotypes, where several different genes may cause the disease, but a single mutation in each case or family has a large effect.

Some doubts have been raised about the ability of systems to predict candidates for complex polygenic diseases such as T2D where multiple genes interact to create a permissive gene pool for disease genesis. The candidate gene prediction systems did prune the genome in favour of moderately to highly significant SNPs identified by the GWAs under semi-blind testing on a complex polygenic disease. Enrichment Ratios calculated in this study suggest that most of the oligogenic benchmarks have been reasonably good predictors of system performance.

\section{Methods}

\section{Benchmark datasets}

Eight candidate gene prediction systems were assessed on their ability to predict genes involved in T2D by comparison against genes implicated by recent GWAs. Two data sets of T2D-implicated genes were used as the benchmark: a Highly Significant gene set (HS) of 21 genes and a Moderate to Highly significant gene set derived from the WTCCC and DIAGRAM studies (MHWD) of 172 genes $[8,9]$. The HS gene set contained 11 genes which mapped to the chromosomal regions investigated by Tiffin et al. (hereafter Tiffin intervals) [7]. Genes associated with 706 moderately significant SNPs with a frequentist additive pvalue of $<0.001$, good clustering and intact NCBI build 36 reference ids were taken from WTCCC T2D data [8]. SNPs positioned between the 5' UTR and 3' UTR of a known gene structure, 1000 bases upstream of a 5' UTR or 1000 bases downstream of 3' UTR of a known gene were considered to implicate the gene in T2D disease susceptibility. This moderately significant list was combined with the genes from the HS data set to generate the MHWD dataset, yielding 172 genes genome wide of which 61 genes mapped to the Tiffin intervals [7]. 


\section{Data sources for predictions}

The search space available to all eight automated candidate gene prediction systems consisted of 9556 genes in 53 chromosomal loci assessed by Tiffin et al. to be involved in T2D by various linkage and association studies. We matched $96.5 \%$ of all Ensembl gene entries [14] provided to NCBI Entrez ids. All remaining genes were unable to be matched due either to the Ensembl entry having an unknown gene symbol label or because the entry was ambiguous or associated with a redundant gene symbol name entry. Ensembl entries and NCBI id matching was carried out at four levels, in order: approved symbol name, previous symbol names, Uniprot/SwissProt Accession and RefSeq Ids. Data conversion keys for matching between databases were acquired from BioMart [15].

Predictions made by seven candidate gene prediction methods were also obtained from Tiffin et al. [7]. Nine disease-implicated genes were available to the systems as seeds (PPARG, GYS1, IRS1, INS, KCNJ11, ABCC8, SLC2A1, PPARGC1, CAPN10). Two of the genes - PPARG and KCNJ11, are implicated by the highly significant SNPs detected by GWAs but are not in the Tiffin intervals and are thus not included in the search space or benchmark set.

\section{Candidate gene predictions}

The candidate gene predictions for seven of the systems are detailed elsewhere [7]. Briefly, GeneSeeker selected genes from the search space using a Boolean expression based on 14 keywords selected by an expert user [7]. PROSPECTR, DGP and eVOC are Boolean classifiers which require only the search space as input. G2D and Gentrepid in $a b$ initio mode, also only require the search space. POCUS potentially only needs the search space as input, but this was restricted to the seven best supported intervals of the 53 available, as judged by the POCUS team. SUSPECTS and Gentrepid, in known-disease-gene mode, used the nine known disease genes associated with the phenotype as seeds. SUSPECTS additionally draws on predictions from the PROSPECTR Boolean classifier.

Gentrepid predictions are discussed in detail here for the first time. Gentrepid implements two different modules to derive predictions: CPS - a systems biology method; and $\mathrm{CMP}$ - a method that associates phenotypes with particular domains. CPS and CMP can be used in two input modes: using known disease genes as a seed or using only the search space ( $a b$ initio mode).

In known-disease-gene input mode, CPS searches all pathway and interaction data in BioCarta [16], KEGG [17] and I2D (formerly OPHID) [10] to extract all genes associated with the disease gene, and then filters this list against implicated loci. Genes are ranked based on the total number of genes implicated in the pathway. For example, if two known disease gene seeds and three genes in the loci being investigated are found in the same pathway, the pathway is given a rank of five against the phenotype. CMP parses the protein sequences of the known disease genes associated with the phenotype into domains using the Pfam library of Hidden Markov Models (HMMs) [18] and then retrieves any other genes with related domain content from the genome. A score between 0 and 1 is generated reflecting the candidate gene's similarity to a known disease gene [4]. The same nine disease genes and 53 chromosomal cytogenetic bands were used by Gentrepid as per Tiffin et al..

In $a b$ initio mode, Gentrepid can make predictions in the absence of known disease genes if two or more loci are provided as input. Gentrepid's CPS $a b$ initio method is based on the premise that pathways whose genes are more prevalent within disease-implicated loci (chromosomal regions) compared to the entire genome have a higher probability of involvement in the pathoetiology of the disease phenotype of interest. Analogous to the known disease gene mode, pathways are ranked by the number of loci involved. The CMP $a b$ initio method searches for enrichment of domains in the loci with respect to the genome and ranks genes based on the statistical significance of the domain enrichment (equations 2 and 4 in [4] where $m n$ is replaced by $\Sigma$ - the total number of genes in the intervals examined).

For each input mode, a final list of predictions is made by consolidating all predictions from both the CMP and CPS modules.

\section{Metrics for comparisons}

Systems were compared using three metrics: Enrichment Ratio, Sensitivity and Specificity. The Enrichment Ratio calculations were calculated as below [4]:

$$
\text { EnrichmentRatio }=\frac{T P /(T P+F P)}{\sum \text { genes } \text { implicated } / \sum \text { genes all }}
$$

The denominator was obtained by dividing the number of T2D implicated genes by the total number of genes within all surveyed chromosomal regions.

Sensitivity and Specificity were calculated as below:

$$
\text { Sensitivity }=\frac{T P}{(T P+F N)}
$$




$$
\text { Specificity }=\frac{T N}{(T N+F P)}
$$

$\mathrm{TP}$ is the number of true positives, FP is the number of false positives, $\mathrm{TN}$ is the number of true negatives and $\mathrm{FN}$ is the number of false negatives. Sensitivity is the proportion of true positives among all disease genes in the chromosomal regions. Specificity is the proportion of true negatives among genes not associated with the disease in chromosomal regions. Confidence intervals were estimated using the method of Newcombe [19] implemented using the CIcalculator software [20].

\section{List of abbreviations used}

T2D: Type II diabetes; WTCCC: Welcome Trust Case Control Consortium; DIAGRAM: Genetics Replication and Meta-analysis Consortium; SNP: Single nucleotide polymorphism; GWA: Genome wide association studies; HPRD: Human Protein Reference Database; BIND: Biomolecular Interaction Network Database; DGP: Disease Gene Prediction; PROSPECTR: PRiOrization by Sequence and Phylogenetic Extent of CandidaTe Regions; OMIM: Online Mendelian Inheritance in Man; OPHID: Online Predicted Human Interaction Database; KEGG: Kyoto Encyclopedia of Genes and Genomes; GO: Gene Ontology; MeSH: Medical Subject Headings; HS: Highly Significant gene set; MHWD: Moderate to Highly gene set derived from WTCCC and DIAGRAM studies; ER: Enrichment Ratio; S: Sensitivity; TP: true positives; FP: false positives; TN: true negatives; FN: false negatives.

\section{Competing interests}

The authors declare that they have no competing interests.

\section{Authors' contributions}

MW design, concept and oversight of study, and manuscript author. ET manuscript author, and data generation for study. JL implementation, construction and maintenance of database. SB additional data generation, figures and manuscript preparation. DF Genetics consultant. All authors read and approved the final manuscript.

\section{Additional material}

\section{Additional file 1}

Additional unprioritized MHWD matches. Additional unprioritized MHWD matches data.

Click here for file

[http://www.biomedcentral.com/content/supplementary/14712105-10-S1-S69-S1.txt]

\section{Acknowledgements}

The authors wish to acknowledge funding from the Ronald Geoffrey Arnott Foundation.

This article has been published as part of BMC Bioinformatics Volume 10 Supplement I, 2009: Proceedings of The Seventh Asia Pacific Bioinformatics Conference (APBC) 2009. The full contents of the supplement are available online at http://www.biomedcentral.com/ |47| $-2105 / 10$ ? issue=SI

\section{References}

I. Gusella JF, Wexler NS, Conneally PM, Naylor SL, Anderson MA, Tanzi RE, Watkins PC, Ottina K, Wallace MR, Sakaguchi AY, Young AB, Shoulson I, Bonilla E, Martin JB: A Polymorphic DNA Marker Genetically Linked to Huntingtons-Disease. Nature 1983, 306(5940):234-238.

2. Hamosh A, Scott AF, Amberger J, Bocchini C, Valle D, McKusick VA Online Mendelian Inheritance in Man (OMIM), a knowledgebase of human genes and genetic disorders. Nucleic Acids Research 2002, 30:52-55.

3. Turner FS, Clutterbuck DR, Semple CAM: POCUS: mining genomic sequence annotation to predict disease genes. Genome Biology 2003, 4(I I):R75.

4. George RA, Liu JY, Feng LL, Bryson-Richardson RJ, Fatkin D, Wouters $M A$ : Analysis of protein sequence and interaction data for candidate disease gene prediction. Nucleic Acids Research 2006, 34(19): el 30.

5. Franke L, van Bakel H, Fokkens L, de Jong ED, Egmont-Petersen $M$, Wijmenga C: Reconstruction of a functional human gene network, with an application for prioritizing positional candidate genes. American Journal of Human Genetics 2006, 78(6): $1011-1025$

6. Motulsky AG: Genetics of complex diseases. J Zhejiang Univ Sci B 2006, 7(2):167-8.

7. Tiffin N, Adie E, Turner F, Brunner HG, van Driel MA, Oti M, LopezBigas N, Ouzounis C, Perez-Iratxeta C, Andrade-Navarro MA, Adeyemo A, Patti ME, Semple CAM, Hide W: Computational disease gene identification: a concert of methods prioritizes type 2 diabetes and obesity candidate genes. Nucleic Acids Research 2006, 34( I 0):3067-308I.

8. Wellcome Trust Case Control Consortium: Genome-wide association study of 14,000 cases of seven common diseases and 3,000 shared controls. Nature 2007, 447(7/4566I-678 [http:// dx.doi.org/10.1038/nature05911].

9. Zeggini E, Scott LJ, Saxena R, Voight BF, Marchini JL, Hu T, de Bakker PIW, Abecasis GR, Almgren P, Andersen G, Ardlie K, Bostrom KB, Bergman RN, Bonnycastle LL, Borch-Johnsen K, Burtt NP, Chen H, Chines PS, Daly MJ, Deodhar P, Ding CJ, Doney ASF, Duren WL, Elliott KS, Erdos MR, Frayling TM, Freathy RM, Gianniny L, Grallert H, Grarup N, Groves C], Guiducci C, Hansen T, Herder C, Hitman GA, Hughes TE, Isomaa $B$, Jackson AU, Jorgensen $T$, Kong $A$, Kubalanza $K$, Kuruvilla FG, Kuusisto J, Langenberg C, Lango H, Lauritzen T, Li Y, Lindgren CM, Lyssenko V, Marvelle AF, Meisinger C, Midthjell K, Mohlke KL, Morken MA, Morris AD, Narisu N, Nilsson P, Owen KR, Palmer CNA, Payne F, Perry JRB, Pettersen E, Platou C, Prokopenko I, Qi L, Qin L, Rayner NW, Rees M, Roix J], Sandbaek A, Shields B, Sjogren M, Steinthorsdottir V, Stringham HM, Swift AJ, Thorleifsson G, Thorsteinsdottir U, Timpson NJ, Tuomi T, Tuomilehto J, Walker M, Watanabe RM, Weedon MN, Willer CJ, Illig T, Hveem K, Hu FB, Laakso M, Stefansson K, Pedersen O, Wareham NJ, Barroso I, Hattersley AT, Collins FS, Groop L, McCarthy MI, Boehnke M, Altshuler $D$ : Meta-analysis of genome-wide association data and largescale replication identifies additional susceptibility loci for type 2 diabetes. Nat Genet 2008, 40(5):638-645.

10. Brown $K R$, Jurisica I: Online predicted human interaction database. Bioinformatics 2005, 2 I (9):2076-2082.

II. Ramani AK, Bunescu RC, Mooney RJ, Marcotte EM: Consolidating the set of known human protein-protein interactions in preparation for large-scale mapping of the human interactome. Genome Biology 2005, 6(5):R40.

12. Adie EA, Adams RR, Evans KL, Porteous DJ, Pickard BS: Speeding disease gene discovery by sequence based candidate prioritization. BMC Bioinformatics 2005, 6:55.

13. van Driel MA, Cuelenaere K, Kemmeren PPCW, Leunissen JAM, Brunner HG, Vriend G: GeneSeeker: extraction and integra- 
tion of human disease-related information from web-based genetic databases. Nucleic Acids Res 2005, 33(Web Server issue):W758-W76I.

14. Birney E, Andrews D, Caccamo M, Chen Y, Clarke L, Coates G, Cox T, Cunningham F, Curwen V, Cutts T, Down T, Durbin R, FernandezSuarez XM, Flicek P, Graf S, Hammond M, Herrero J, Howe K, lyer V, Jekosch K, Kahari A, Kasprzyk A, Keefe D, Kokocinski F, Kulesha E, London D, Longden I, Melsopp C, Meidl P, Overduin B, Parker A, Proctor G, Prlic A, Rae M, Rios D, Redmond S, Schuster M, Sealy I, Searle S, Severin J, Slater G, Smedley D, Smith J, Stabenau A, Stalker J, Trevanion S, Ureta-Vidal A, Vogel J, White S, Woodwark C, Hubbard TJP: Ensembl 2006. Nucleic Acids Research 2006, 34:D556-D56I.

15. Kasprzyk A, Keefe D, Smedley D, London D, Spooner W, Melsopp C, Hammond M, Rocca-Serra P, Cox T, Birney E: A generic system for fast and flexible access to biological data. Genome Research 2004, I 4:160-169.

16. BioCarta [http://www.biocarta.com]

17. Kanehisa M, Goto S, Kawashima S, Okuno Y, Hattori M: The KEGG resource for deciphering the genome. Nucleic Acids Research 2004, 32:D277-D280.

18. Bateman A, Birney E, Cerruti L, Durbin R, Etwiller L, Eddy SR, Griffiths-Jones S, Howe KL, Marshall M, Sonnhammer ELL: The Pfam Protein Families Database. Nucleic Acids Research 2002, 30:276-280.

19. Newcombe RG: Improved confidence intervals for the difference between binomial proportions based on paired data. Statistics in Medicine 1998, I 7(22):2635-2650.

20. Clcalculator software. . http://www.pedro.fhs.usyd.edu.au/calculator.html

21. Adie EA, Adams RR, Evans KL, Porteous DJ, Pickard BS: SUS PECTS: enabling fast and effective prioritization of positional candidates. Bioinformatics 2006, 22(6):773-774.

22. Mulder NJ, Apweiler R, Attwood TK, Bairoch A, Bateman A, Binns D, Bork P, Buillard V, Cerutti L, Copley R, Courcelle E, Das U, Daugherty L, Dibley M, Finn R, Fleischmann W, Gough J, Haft D, Hulo N, Hunter S, Kahn D, Kanapin A, Kejariwal A, Labarga A, LangendijkGenevaux PS, Lonsdale D, Lopez R, Letunic I, Madera M, Maslen J, McAnulla C, McDowall J, Mistry J, Mitchell A, Nikolskaya AN Orchard S, Orengo C, Petryszak R, Selengut JD, Sigrist CJA, Thomas $\mathrm{PD}$, Valentin F, Wilson D, Wu CH, Yeats C: New developments in the InterPro database. Nucleic Acids Research 2007, 35(Database issue):D224-D228.

23. Ashburner M, Ball CA, Blake JA, Botstein D, Butler $H$, Cherry JM, Davis AP, Dolinski K, Dwight SS, Eppig IT, Harris MA, Hill DP, IsselTarver L, Kasarskis A, Lewis S, Matese JC, Richardson JE, Ringwald M, Rubin GM, Sherlock G: Gene Ontology: tool for the unification of biology. Nature Genetics 2000, 25:25-29.

24. Oti M, Snel B, Huynen MA, Brunner HG: Predicting disease genes using protein-protein interactions. Journal of Medical Genetics 2006, 43(8):69|-8.

25. Badano JL, Katsanis N: Beyond Mendel: An evolving view of human genetic disease transmission. Nature Reviews Genetics 2002, 3(I 0):779-789.

26. Jimenez-Sanchez G, Childs B, Valle D: Human disease genes. Nature 200I, 409(6822):853-855

27. Dudley AM, Janse DM, Tanay A, Shamir R, Church GM: A global view of pleiotropy and phenotypically derived gene function in yeast. Molecular Systems Biology 2005:2005.000I.

28. Ohya Y, Sese J, Yukawa M, Sano F, Nakatani Y, Saito TL, Saka A, Fukuda T, Ishihara S, Oka S, Suzuki G, Watanabe M, Hirata A, Ohtan M, Sawai H, Fraysse N, Latge JP, Francois JM, Aebi M, Tanaka S, Muramatsu S, Araki H, Sonoike K, Nogami S, Morishita S: High-dimensional and large-scale phenotyping of yeast mutants. Proceedings of the National Academy of Sciences of the United States of America 2005, 102(52): $19015-19020$

29. Freudenberg J, Propping P: A similarity-based method for genome-wide prediction of disease-relevant human genes. Bioinformatics 2002, I8:SIIO-SII5.

30. Tiffin N, Kelso JF, Powell AR, Pan H, Bajic VB, Hide WA: Integration of text- and data-mining using ontologies successfully selects disease gene candidates. Nucleic Acids Research 2005 33(5): $1544-1552$.

31. Lopez-Bigas N, Ouzounis CA: Genome-wide identification of genes likely to be involved in human genetic disease. Nucleic Acids Research 2004, 32(10):3108-3114.
32. Perez-Iratxeta C, Bork $P$, Andrade MA: Association of genes to genetically inherited diseases using data mining. Nature Genetics 2002, 3 I (3):316-319.

33. GeneSeeker web tool [http://www.cmbi.ru.nl/geneseeker]

34. Alfarano C, Andrade CE, Anthony K, Bahroos N, Bajec M, Bantoft K, Betel D, Bobechko B, Boutilier K, Burgess E, Buzadzija K, Cavero R, D'Abreo C, Donaldson I, Dorairajoo D, Dumontier MJ, Dumontier MR, Earles V, Farrall R, Feldman H, Garderman E, Gong Y, Gonzaga R, Grytsan V, Gryz E, Gu V, Haldorsen E, Halupa A, Haw R, Hrvojic $A$, Hurrell L, Isserlin R, Jack F, Juma F, Khan A, Kon T, Konopinsky S, Le V, Lee E, Ling S, Magidin M, Moniakis J, Montojo J, Moore S, Muskat B, Ng I, Paraiso JP, Parker B, Pintilie G, Pirone R, Salama J], Sgro S, Shan T, Shu Y, Siew J, Skinner D, Snyder K, Stasiuk R, Strumpf D, Tuekam B, Tao S, Wang Z, White M, Willis R, Wolting C, Wong S, Wrong A, Xin C, Yao R, Yates B, Zhang S, Zheng K, Pawson T, Ouellette BFF, Hogue CWV: The Biomolecular Interaction Network Database and related tools 2005 update. Nucleic Acids Research 2005, 33:D4I8-D424.

35. Joshi-Tope G, Gillespie M, Vastrik I, D'Eustachio P, Schmidt E, de Bono B, Jassal B, Gopinath GR, Wu GR, Matthews L, Lewis S, Birney $E$, Stein L: Reactome: a knowledgebase of biological pathways. Nucleic Acids Research 2005, 33:D428-D432.

36. Peri S, Navarro JD, Amanchy R, Kristiansen TZ, Jonnalagadda CK, Surendranath V, Niranjan V, Muthusamy B, Gandhi TKB, Gronborg M, lbarrola N, Deshpande N, Shanker K, Shivashankar HN, Rashmi BP, Ramya MA, Zhao ZX, Chandrika KN, Padma N, Harsha HC, Yatish AJ, Kavitha MP, Menezes M, Choudhury DR, Suresh S, Ghosh N, Saravana R, Chandran S, Krishna S, Joy M, Anand SK, Madavan V, Joseph A, Wong GW, Schiemann WP, Constantinescu SN, Huang LL, Khosravi-Far R, Steen H, Tewari M, Ghaffari S, Blobe GC, Dang CV, Garcia JGN, Pevsner J, Jensen ON, Roepstorff P, Deshpande KS, Chinnaiyan AM, Hamosh A, Chakravarti A, Pandey A: Development of human protein reference database as an initial platform for approaching systems biology in humans. Genome Research 2003, I3( I 0):2363-237I.

37. Ito T, Chiba T, Ozawa R, Yoshida M, Hattori M, Sakaki Y: A comprehensive two-hybrid analysis to explore the yeast protein interactome. Proceedings of the National Academy of Sciences of the United States of America 200I, 98(8):4569-4574.

38. Uetz P, Giot L, Cagney G, Mansfield TA, Judson RS, Knight JR, Lockshon D, Narayan V, Srinivasan M, Pochart P, Qureshi-Emili A, Li Y, Godwin B, Conover D, Kalbfleisch T, Vijayadamodar G, Yang MJ, Johnston M, Fields S, Rothberg JM: A comprehensive analysis of protein-protein interactions in Saccharomyces cerevisiae. Nature 2000, 403(6770):623-627.

39. Gavin AC, Bosche M, Krause R, Grandi P, Marzioch M, Bauer A, Schultz J, Rick JM, Michon AM, Cruciat CM, Remor M, Hofert C, Schelder M, Brajenovic M, Ruffner H, Merino A, Klein K, Hudak M, Dickson D, Rudi T, Gnau V, Bauch A, Bastuck S, Huhse B, Leutwein C, Heurtier MA, Copley RR, Edelmann A, Querfurth E, Rybin V, Drewes G, Raida M, Bouwmeester T, Bork P, Seraphin B, Kuster B, Neubauer G, Superti-Furga G: Functional organization of the yeast proteome by systematic analysis of protein complexes. Nature 2002, 4I 5(6868): | 4 | - I 47.

40. Ho Y, Gruhler A, Heilbut A, Bader GD, Moore L, Adams SL, Millar A, Taylor P, Bennett K, Boutilier K, Yang LY, Wolting C, Donaldson I, Schandorff S, Shewnarane J, Vo M, Taggart J, Goudreault M, Muskat B, Alfarano C, Dewar D, Lin Z, Michalickova K, Willems AR, Sassi H, Nielsen PA, Rasmussen KJ, Andersen JR, Johansen LE, Hansen LH, Jespersen $H$, Podtelejnikov A, Nielsen E, Crawford J, Poulsen V, Sorensen BD, Matthiesen J, Hendrickson RC, Gleeson F, Pawson T, Moran MF, Durocher D, Mann M, Hogue CWV, Figeys D, Tyers M: Systematic identification of protein complexes in Saccharomyces cerevisiae by mass spectrometry. Nature 2002, 4I5(6868): | $80-183$

4I. DGP web tool [http://cgg.ebi.ac.uk/services/dgp] 